PAPER

\title{
Quantified measurement of activity provides insight into motor function and recovery in neurological disease
}

\author{
M E Busse, O R Pearson, R Van Deursen, C M Wiles
}

J Neurol Neurosurg Psychiatry 2004;75:884-888. doi: 10.1136/jnnp.2003.020180

See end of article for authors' affiliations .....................

Correspondence to: Dr R van Deursen, Research Centre for Clinical Kinaesiology, Department of Physiotherapy Education, University of Wales College of Medicine, Heath Park, Cardiff 'CF14 4XN, UK; vandeursenr@ cardiff.ac.uk

Received 13 April 2003

In revised form

16 September 2003

Accepted

20 September 2003

\begin{abstract}
Background: A direct quantitative measurement of locomotor activity in an individual's own environment over an extended period may help in evaluating the impact of impairments in neurological disorders.

Objective: To investigate the reliability and validity of activity monitoring in neurological patients and healthy subjects.

Methods: Initial reliability studies were completed on 10 healthy subjects and 10 mobility restricted neurological patients. Validity was investigated using 7 days of ambulatory monitoring with the Step Watch $^{\text {TM }}$ step activity monitor, laboratory based measures of gait and the Rivermead Mobility Index (RMI) in 10 patients with multiple sclerosis, 10 with Parkinson's disease, and 10 with a primary muscle disorder. Additionally, 30 healthy subjects participated in the study. Two clinical illustrations of ambulatory monitoring are provided.

Results: The mean (range) right step count of 7 days of monitoring in both healthy and neurological patients proved a reliable measure of activity (intra-class correlations 0.89 and 0.86 respectively). The 7 day mean (range) right step count was 5951 (2886-9955) in healthy subjects, $3818(1611-5391)$ in patients with Parkinson's disease, 3003 (716-5302) in those with muscular disorders, and 2985 (6895340 ) in those with multiple sclerosis. A moderate correlation was noted between 7 day mean step count and gait speed $(r=0.45, p=0.01)$ in the grouped neurological patients but not the $\operatorname{RMl}\left(r_{s}=0.3, p=0.11\right)$. Conclusion: Ambulatory monitoring provides a reliable and valid measure of activity levels. Neurological patients, living independently, demonstrate lower activity levels than healthy matched controls. Ambulatory monitoring as an outcome measure has potential for improving the evaluation of ambulation and providing insight into participation.
\end{abstract}

reliability of activity monitoring in stroke patients indicates that there is good potential for their use in the quantification of home/community based activity levels. ${ }^{3}$ This study further investigates reliability and validity and illustrates the application of long term ambulatory monitoring in a neurological population with the aim of providing insight into motor function and recovery in neurological disease.

\section{METHODS}

\section{Techniques}

Steps were measured with the Step Watch ${ }^{\mathrm{TM}}$ (Cymatech, Seattle, Washington, USA) step activity monitor (SAM), a sealed, waterproof, micro-processor controlled device weighing $65 \mathrm{~g}$ (height $6.5 \mathrm{~cm}$, width, $5 \mathrm{~cm}$, and depth $1.5 \mathrm{~cm}$ ). The monitor is attached to the right lower limb immediately above the lateral malleolus of the ankle with two elastic attachment straps (see fig 1). The SAM records step count sub-totals (see fig 2), overall activity levels, and patterns of activity and rest in programmable time intervals over extended monitoring periods. The SAM is programmed via an infrared optical interface prior to the monitoring period with sensitivity settings appropriate to the height, cadence, and gait speed of the individual being monitored. The settings are verified by visual inspection during a test trial where a light on the monitor is programmed to blink each time a step is counted for the first 30 steps taken by the individual. Activity measures produced are daily step counts, sustained activity measures and peak activity indices.

Abbreviations: EDSS, Expanded Disability Status Scale; GBS, GuillainBarré syndrome; ICF, International Classification of Functioning; MD, muscle disorder; MS, multiple sclerosis; PD, Parkinson's disease; RMI, Rivermead Mobility Index; SAM, step activity monitor style of the subject. These devices have been used successfully in populations with gait asymmetry. ${ }^{7}$ The accuracy and 
Sustained activity measures are derived via the activity monitoring software by extracting the maximum number of steps achieved during any continuous interval of specified duration (for example, 20, 30, or 60 minutes); that maximum is divided by the duration of the interval to give the mean steps per minute. The peak activity index represents the highest mean step rate during any $30 \times 1$ minute intervals of the 24 hour period. This differs from the sustained activity measures in that the performance does not occur in a continuous time frame. Levels of inactivity are also produced by the system software. These are presented as the percentage of the total time in a 24 hour period where no steps are recorded. Monitoring levels of inactivity is useful for assessing change in walking and has been used in the clinical cases presented.

Measures of self selected gait speed and function were obtained prior to attachment of the SAM. Gait speed was measured at self selected walking pace over 10 metres in a motion analysis laboratory using digital video technology (including start/stop).${ }^{10}$ RMI was evaluated in all neurological patients. The EDSS was scored by a neurologist for the MS patients.

\section{Subjects and protocol}

This study received prior ethical approval from the local research ethics committee. All participants signed an informed consent document prior to initiation of the study.

\section{Reliability}

A convenience sample of 10 healthy subjects and 10 ambulant neurological patients were recruited. Impairments due to different pathologies were included, namely motor neurone disease $(\mathrm{n}=2)$, hereditary spastic paraparesis $(\mathrm{n}=1)$, peripheral neuropathy $(\mathrm{n}=1)$, myasthenia gravis $(n=2)$, myositis $(n=1)$, muscular dystrophy $(n=1)$, thyroid myopathy $(n=1)$, and Parkinson's disease (PD) $(n=1)$. The over-riding characteristic of all the neurological patients was that they had restricted walking mobility; however, all were able to walk more than a distance of 10 metres independently with use of a walking aid (but no standby assistance). Patients did not receive any physiotherapy treatment during or between the measurement phases.

All subjects participated in two 7 day periods of monitoring, with at least $1-3$ weeks between each period. The SAM was attached to the lateral aspect of the right ankle and recorded activity level by counting steps taken per minute continuously throughout the measurement period. All subjects wore the device for 24 hours every day and only removed it for bathing. Subjects were asked to report any instances during which the device had to be removed.

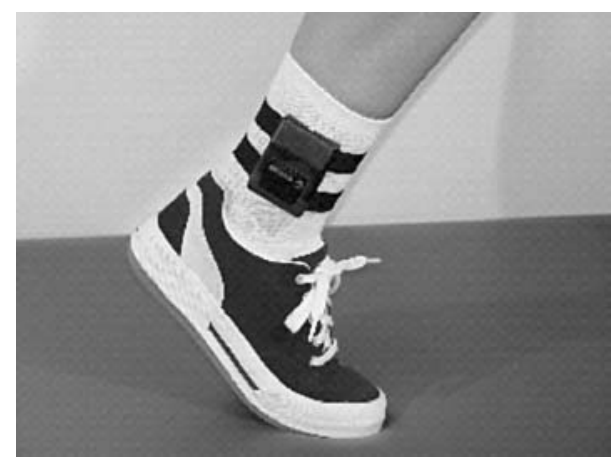

Figure 1 SAM as applied to the right ankle of the subject.

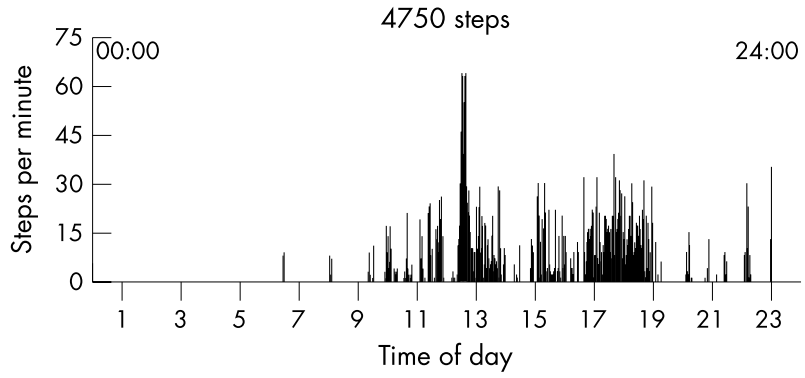

Figure 2 Illustrative download of graphical output from for one day of step activity monitoring in a healthy subject. The $x$ axis represents the 24 hour day and the height of each bar represents number of steps per minute. The shaded bands depicted are set for less that 15 steps $/ \mathrm{min}$ indicating low levels of activity (dark grey), between 15 and 40 steps/ min indicating medium levels of activity (light grey) and greater than 40 steps/min indicating high levels of activity (white).

\section{Validity}

A second sample of 30 healthy subjects and 10 patients with $\mathrm{PD}, 10$ with multiple sclerosis (MS), and 10 with a primary muscle disorder (MD) were recruited. All patients were able to walk a distance of 10 metres independently, with the use of a walking aid where necessary. Patients were recruited from general neurological clinics.

The recruited subjects were monitored for seven consecutive days with the SAM. Self selected gait speed and functional status as reflected by the Rivermead Mobility Index (RMI) in its standard form ${ }^{8}$ were recorded prior to the first week of monitoring.

\section{Clinical illustration of ambulatory monitoring}

A 63 year old male with Guillain-Barré syndrome (GBS) was followed during recovery in hospital and at home, tracking motor recovery over 9 months (four serial measurements). A 42 year old male recovering from a right hemiparetic MS relapse was followed during hospitalisation and following discharge (two serial measurements).

Both patients were monitored with respect to RMI, gait speed and 7 day mean step count (SAM) at each measurement session. The Expanded Disability Status Scale (EDSS) was scored by a neurologist for the patient recovering from an MS relapse. ${ }^{9}$

\section{Data analysis}

\section{Reliability}

Intraclass correlations (ICC) were calculated between individual days of each monitoring period (day to day reliability) as well as between the mean step count for each 7 day period (week to week reliability). Intra-class correlations for the peak activity index (highest mean step rate for $30 \mathrm{~min} /$ day in steps/min) and sustained activity index (maximum number of steps in any continuous period of 20,30, and 60 minutes' duration in mean steps/min) were also calculated. The average coefficient of variation (CV) was calculated as the standard deviation of the measures expressed as a percentage (\%) of the mean, ${ }^{11}{ }^{12}$ in order to ascertain the variation of the sample as necessitated by the dependency of the ICC on the variation of the sample. A scatter plot of week to week reliability was used to inspect for systematic deviations from the identity line. ${ }^{13}$

\section{Validity}

Correlation coefficients between the 7 day mean step count, gait speed, and functional score (RMI) were calculated. Independent $t$ tests were used to assess for differences in 7 day mean step counts between the age and sex matched 
healthy subjects and the separate neurological patient groups.

\section{RESULTS}

All subjects completed all the required phases of monitoring. The SAM was well tolerated in the required ankle attachment position. There were no reports of complications or discomfort. All subjects were compliant in continuous wearing of the monitor throughout the monitoring period. This was confirmed by visual inspection of the data. Subject and matched control details, mean right step counts, gait speed, RMI, and EDSS where applicable for the reliability and validity studies are presented in table 1 .

\section{Reliability of step counts}

The 7 day mean step count in both healthy $($ ICC $=0.89)$ and neurological patients (ICC $=0.86$ ) provided a reliable measure of daily activity. Day to day variability in both healthy individuals and those with neurological impairment was relatively high as reflected by the CV. The average CV for day to day step count in healthy subjects was $28 \%$ and in neurological patients $30 \%$ (table 2 ). The average CV for week to week measures in healthy subjects was $8.8 \%$ and in neurological patients $12 \%$ (see table 2 ). Fig 3 depicts the scatter plot of week to week reliability in neurological patients using the 7 day mean step count. ${ }^{14}$

\section{Reliability of other activity indices}

The mean peak activity index, which represents the highest mean step rate during any 30 discrete 1 minute intervals of the 24 hour period, was 59 steps/min in healthy subjects and 36 steps/min in neurological patients. The mean (SD) peak activity indices and the intraclass correlation coefficients for the reliability are presented in table 3. Sustained activity represents continuous activity in steps/min maintained over the entire specified time period. Mean sustained activity over 60 minute intervals was 41 steps/min in healthy subjects and $17 \mathrm{steps} / \mathrm{min}$ in neurological patients. Greater variability for the sustained activity indices in healthy subjects was apparent.

\section{Validity}

There were significant differences $(p=0.001)$ for mean 7 day step count in all the separately matched patient groups compared with healthy control subjects: (mean difference 2529,3944 , and 2365; 95\% confidence interval (CI) 1292 to 3763, 2366 to 5521 , and 861 to 3868 for PD, MS, and MD

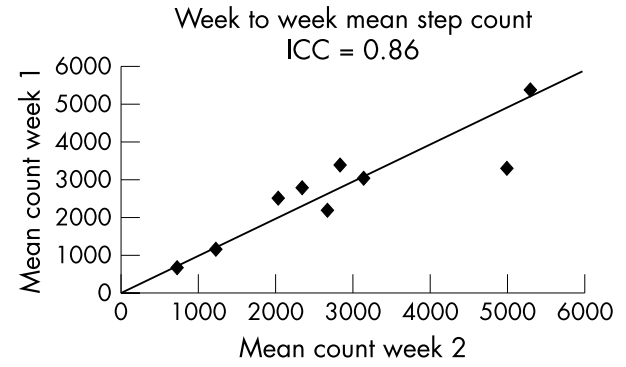

Figure 3 Scatter plot of week to week reliability in neurological patients using the 7 day mean step count.

respectively). Overall 7 day mean step counts were substantially lower in neurological patients compared with healthy subjects (table 1), with overall difference in mean 7 day step count of 2945. Similarly, peak activity and sustained activity measures were lower in patients than healthy subjects. There was negative, non-significant correlation between gait speed and 7 day mean step counts $(r=-0.17, p=0.37)$ in the healthy subjects $(n=30)$. A moderate correlation was noted between gait speed and 7 day mean step counts $(r=0.45$, $\mathrm{p}=0.01$ ) in the grouped neurological patients but not between 7 day mean step counts and RMI $\left(r_{s}=0.3\right.$, $\mathrm{p}=0.11)$.

\section{Clinical illustration of responsiveness}

The GBS patient's mean daily step count increased from 303 $(\mathrm{RMI}=6)$ to $649(\mathrm{RMI}=8)$ to $1914(\mathrm{RMI}=12)$ to 2392 $(\mathrm{RMI}=13)$ over a 9 month period (four serial measurements) (fig 4) and time spent involved in no activity decreased from $97.4 \%$ to $95.2 \%$ to $85.4 \%$ to $84 \%$. Peak activity index increased from 13.5 to 19.4 to 35.5 to 49.6 steps $/ \mathrm{min}$.

The MS patient's 7 day mean step count while recovering from a right hemiparetic relapse increased from 836 $(\mathrm{RMI}=9)$ to $4015(\mathrm{RMI}=14)$ over a 2 month period, while the EDSS score decreased from 6.0 to 4.0. A score of 6.0 on the EDSS means that the individual requires unilateral assistance to walk at least 100 metres, while a score of 4.0 on the EDSS means that the individual is restricted but able to walk without an aid over a distance of at least 500 metres. Time spent involved in no activity decreased from $90.3 \%$ to $72.9 \%$, and peak activity index increased from 20.2 to 37.2 steps/min.

Table 1 Descriptive details of study sample for reliability and validity procedures

\begin{tabular}{|c|c|c|c|c|}
\hline Subject details & $\begin{array}{l}\text { Mean age, } \\
\text { years (SD) }\end{array}$ & $\begin{array}{l}\text { Mean } 7 \text { day step } \\
\text { count (range) }\end{array}$ & $\begin{array}{l}\text { Mean gait speed, } \\
\mathrm{m} / \mathrm{s} \text { (SD) }\end{array}$ & $\begin{array}{l}\text { Median RMI } \\
\text { (range; 0-15) }\end{array}$ \\
\hline \multicolumn{5}{|l|}{ Reliability study } \\
\hline Healthy subjects $(n=10)(6$ male $)$ & $43.3(18.9)$ & $6520(3613-10002)$ & $1.49(0.26)$ & \\
\hline $\begin{array}{l}\text { Neurology patients ( } \mathrm{n}=10)(5 \text { male) } \\
\text { Validity study }\end{array}$ & $59.4(13.4)$ & 2961 (716-5301) & $0.82(0.38)$ & $12(8-14)$ \\
\hline MS patients $(\mathrm{n}=10)(0$ male $)$ & $37.9(10.1)$ & $2985(689-5340)$ * & $1.06(0.43)$ & $13(9-15)$ \\
\hline $\begin{array}{l}\text { Matched MS healthy subjects } \\
(n=10)\end{array}$ & $37.5(12.6)$ & $6929(4347-10002)$ & $1.33(0.21)$ & \\
\hline PD patients $(n=10)(7$ male $)$ & $67.1(8.2)$ & $3818(1611-5391)$ * & $0.99(0.16)$ & $13(9-15)$ \\
\hline $\begin{array}{l}\text { Matched PD healthy subjects }(n=10) \\
\text { MD patients }(n=10)(6 \text { male) }\end{array}$ & $\begin{array}{l}66.8(10.8) \\
52.1(12.5)\end{array}$ & $\begin{array}{l}6347(4703-9955) \\
3003(716-5302) *\end{array}$ & $\begin{array}{l}1.22(0.23) \\
0.79(0.23)\end{array}$ & $12(9-15)$ \\
\hline $\begin{array}{l}\text { Matched MD healthy subjects } \\
(n=10)\end{array}$ & $49.5(16.7)$ & $5368(2886-7758)$ & $1.37(0.3)$ & \\
\hline Grouped neurology patients $(n=30)$ & $52.4(15.7)$ & 3268 (689-5391) & $0.95(0.3)$ & $13(9-15)$ \\
\hline Healthy subjects $(n=30)$ & $51.3(17.9)$ & $6214(2886-10002)$ & $1.3(0.25)$ & \\
\hline
\end{tabular}

*Indicates significant difference to the matched group $(p=0.001)$.

$M D$, primary muscle disorder; MS, multiple sclerosis; PD, Parkinson's disease. 
Table 2 Daily step count (1-7) in neurological patients $(n=10)$

\begin{tabular}{|c|c|c|c|c|c|c|c|c|c|c|c|c|c|}
\hline \multicolumn{7}{|c|}{ Step count } & \multicolumn{2}{|c|}{$\begin{array}{l}7 \text { day mean step } \\
\text { count }\end{array}$} & \multicolumn{2}{|c|}{ Day to day step count } & \multicolumn{3}{|c|}{ Week to week mean step count } \\
\hline Day 1 & Day 2 & Day 3 & Day 4 & Day 5 & Day 6 & Day 7 & Week A & Week B & $\overline{S D}$ & CV, \% & Mean & SD & $\mathrm{CV}, \%$ \\
\hline 861 & 1135 & 1310 & 1116 & 1706 & 1017 & 1058 & 1171 & 1214 & 327.9 & 27.0 & 1192 & 30 & 2.5 \\
\hline 2959 & 2837 & 3543 & 3164 & 3896 & 2644 & 3832 & 3267 & 4996 & 1884.8 & 37.7 & 4131 & 1222 & 29.6 \\
\hline 1976 & 2903 & 2392 & 2326 & 2262 & 2907 & 2798 & 2509 & 2024 & 336.8 & 16.6 & 2266 & 342 & 15.1 \\
\hline 1592 & 6508 & 2088 & 2453 & 8709 & 7147 & 9079 & 5368 & 5301 & 2819.5 & 53.2 & 5334 & 46 & 0.9 \\
\hline 3673 & 3041 & 3515 & 2641 & 2426 & 2943 & 1194 & 2776 & 2346 & 734.4 & 31.3 & 2561 & 303 & 11.9 \\
\hline 1964 & 2729 & 5232 & 2104 & 2919 & 4043 & 2346 & 3048 & 3140 & 844.0 & 26.9 & 3094 & 65 & 2.1 \\
\hline 3530 & 2733 & 3622 & 3989 & 2828 & 4212 & 2817 & 3390 & 2829 & 765.5 & 27.1 & 3109 & 396 & 12.7 \\
\hline 617 & 658 & 769 & 696 & 617 & 693 & 539 & 655 & 716 & 171.4 & 23.9 & 685 & 43 & 6.3 \\
\hline
\end{tabular}

Mean, standard deviation (SD) and co-efficient of variation (CV) for 1 week of monitoring and the mean, SD and CV of the mean 7 day step count for weeks 1 and 2 are shown.

\section{DISCUSSION}

The ideal assessment of an individual's walking (what they actually do) might include measures of distance, number of paces, and assistance used over a representative time period in the community. Most current assessments examine what an individual does or believes they can do, in a particular, usually brief, test situation. In these circumstances, environment, motivation for the test, and a range of transient factors may make the test result a very uncertain index of everyday walking. At the very least, the relationship between brief tests of walking and normal walking in the community requires further exploration. As yet, there is no clear standard for the measurement of actual walking in the community setting. ${ }^{15}$

The current study shows that it is possible to measure total number of paces over 7 days in healthy subjects and neurological patients unobtrusively and reliably. In view of the variability identified in activity measurement of single days and the relatively high associated mean coefficient of variation of $30 \%$, the mean of 7 days of monitoring is recommended as a reliable and valid measure of activity in all subjects.

The peak and sustained activity indices allow for objective assessment of the pattern and intensity of activity in all subjects, with valuable potential for assessment of outcome following intervention. As well as having higher mean 7 day step counts, healthy subjects have higher but more variable sustained activity levels and higher mean peak levels than neurological patients. The latter have more variable and lower mean peak activity measures but more consistent sustained activity indices. Fatigue and related impairments may limit neurological patients by restricting the actual number of steps that they are able to maintain for an extended period of time. While activity levels generally are, as expected, lower in patients, the overall consistency of sustained activity levels might indicate a reduced ability to vary activity level because of a wide range of neurological and/or behavioral impairments and modifications. It is notable that during recovery, individuals begin to demonstrate improved peak measures with increasing consistency

Table 3 Reliability study

\begin{tabular}{llllll}
\hline \multicolumn{5}{c}{ Activity index, mean (SD) ICC } \\
\cline { 2 - 5 } Study group & Peak & $\begin{array}{l}\text { 20 minute } \\
\text { sustained }\end{array}$ & $\begin{array}{l}30 \text { minute } \\
\text { sustained }\end{array}$ & $\begin{array}{l}60 \text { minute } \\
\text { sustained }\end{array}$ \\
\hline $\begin{array}{l}\text { Healthy subjects } \\
(n=10)\end{array}$ & $59(5) 0.98$ & $53(10) 0.75$ & $49(13) 0.71$ & $41(13) 0.57$ \\
$\begin{array}{l}\text { Neurology } \\
\text { patients }(n=10)\end{array}$ & $36(11) 0.82$ & $26(10) 0.94$ & 22 & $(9) 0.90$ & $17(8) 0.95$ \\
\hline
\end{tabular}

Mean (SD) activity indices in steps/min and intraclass correlation coefficients (ICC) for activity indices. and more variability in sustained activity as they are able to participate more in their own community.

The ability to count every step taken by an individual within his own environment has obvious face validity. In the absence of an accepted gold standard against which to relate a new mobility measure, comparison of a new method with existing measures that are thought to measure a similar construct (mobility) ${ }^{16}$ is appropriate, although as has been pointed out above, quite different aspects of mobility may be measured. Assessment of validity also involves testing whether a measure behaves as hypothesised, thus mobility should differ according to disease progression. As expected, our neurological patients (although all able to walk independently without the aid of another person), demonstrated significantly lower activity levels than healthy matched controls. Moderate correlations found between the 7 day mean step count, gait speed, and lack of any correlation between 7 day mean step count and RMI justify the view that gait speed and RMI, though reliable tests, may not be good indicators of actual walking ability in the community. This highlights the need for further investigation, using long term monitoring, of the methods used by individuals with mobility restrictions to achieve their best functional capacity. The use of a debriefing session after 7 days of monitoring to detail the
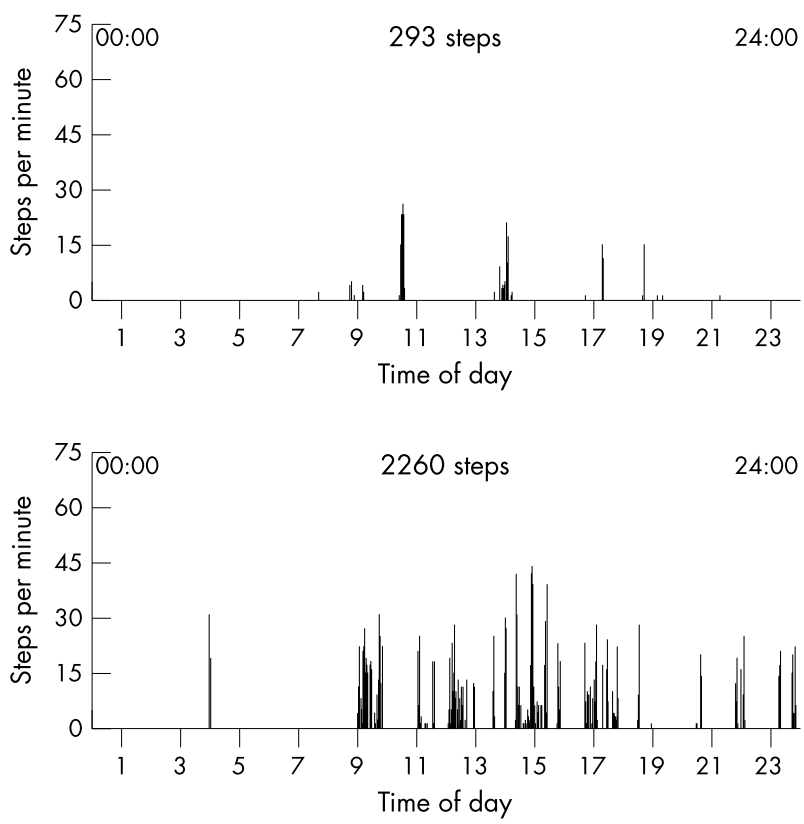

Figure 4 Clinical illustration of ambulatory monitoring using a download of graphical output from 2 days of monitoring in the patient with GBS (first and fourth measurement of four). 
week's activity is currently being investigated. The threshold for independent living is as yet unknown and needs further investigation. The development of a database of 7 day mean step counts and activity levels in healthy subjects and patients with different types and levels of impairment will aid this process.

The two patients studied over time merely illustrate the large scale of potential change found with activity monitoring. Further research in ambulatory monitoring is necessary to clarify the changes to be expected as a result of common therapies or medical interventions and the relationship of such changes (improvement or deterioration) to measures of participation and quality of life.

It should be acknowledged that while it may not be sufficient to rely upon timed tests of walking or functional indices on their own, there remain methodological issues related to long term ambulatory monitoring. The compliance of the subject being monitored (for example, removing the monitor), the possibility of measurement reactivity, and the impact of the environment on activity is relevant to data interpretation. There is potential for missed step counts or excessive step counts, although the sensitivity settings mean that different styles of gait can be accommodated for. There could also be problems with accuracy during stair climbing and during the completion of indoor activities requiring small steps with little distance covered, although preliminary studies have found the monitor to be $98 \%$ accurate compared with hand tallied counts during 6 minute walk tests on level surfaces, ${ }^{3}$ with a $96.2 \%$ accuracy during stair ascent and descent. ${ }^{4}$ The issue of wheelchair use and its relationship to overall mobility in more disabled individuals is clearly an area that requires further study in evaluating the link between impaired walking and participation.

The use of motion sensors has been advocated for comprehensive quantification of mobility. ${ }^{17}$ Long term activity monitoring of step counts and activity patterns combined with measures of gait parameters has the potential to provide a more comprehensive and valid description of activity. Levels of activity in the home environment can now be quantified and this may aid decision making in the process of neurological rehabilitation. Such evaluations of walking activity may also be relevant to a more objective assessment of interventions (such as medication and physiotherapy) that purport to improve a patient's walking. ${ }^{18}$

\section{ACKNOWLEDGMENTS}

This study was funded by the Wales Office for Research and Development (DTA 00_2_008). We would like to acknowledge the staff in the Department of Neurology, University Hospital of Wales and the Research Centre for Clinical Kinaesiology, University of Wales College of Medicine, Cardiff. The assistance of the healthy subjects and neurological patients is also gratefully acknowledged.

\section{Authors' affiliations}

M E Busse, R Van Deursen, Departments of Physiotherapy Education, University of Wales College of Medicine, Cardiff, UK

O R Pearson, C M Wiles, Department of Medicine (Neurology), University of Wales College of Medicine, Cardiff, UK

Competing interests: none declared

\section{REFERENCES}

1 Dahl TH. International classification of functioning, disability and health: an introduction and discussion of its potential impact on rehabilitation services and research. J Rehabil Med 2002;34:201-4.

2 May D, Nayak US, Isaacs B. The life-space diary: a measure of mobility in old people at home. Int Rehabil Med 1985;7:182-6.

3 Macko RF, Haeuber E, Shaughnessy M, et al. Microprocessor-based ambulatory activity monitoring in stroke patients. Med Sci Sports Exerc 2002;34:394-9.

4 Coleman KL, Smith DG, Boone DA, et al. Step activity monitor: long-term, continuous recording of ambulatory function. J Rehabil Res Dev 1999;36:8-18.

$5 \mathrm{Ng} \mathrm{AV}$, Kent-Braun JA. Quantitation of lower physical activity in persons with multiple sclerosis. Med Sci Sports Exerc 1997;29:517-23.

6 Shepherd EF, Toloza E, McClung CD, et al. Step activity monitor: increased accuracy in quantifying ambulatory activity. J Orthop Res 1999;17:703-8.

7 Hartsell H, Fitzpatrick D, Brand R, et al. Accuracy of a custom-designed activity monitor: implications for diabetic foot ulcer healing. J Rehabil Res Dev 2002;39:395-400

8 Forlander DA, Bohannon RW. Rivermead Mobility Index: a brief review of research to date. Clin Rehabil 1999;13:97-100

9 Kurtzke JF. Rating neurologic impairment in multiple sclerosis: an expanded disability status scale (EDSS). Neurology 1983;33:1444-52.

10 Van Deursen R, Button K, Lawthom C. Measurement of spatial and temporal gait parameters using a digital camcorder. Gait Posture $2001 ; 14: 128$.

11 Daniel W. Biostatistics: a foundation for analysis in the health sciences, 6th edn. New York: John Wiley \& Sons, 1995.

12 Hazard Munro B. Statistical methods for health care research, 4th edn. Philadelphia: Lippincott Williams \& Wilkins, 2001.

13 Bland JM, Altman DG. A note on the use of the intraclass correlation coefficient in the evaluation of agreement between two methods of measurement. Comput Biol Med 1990;20:337-40.

14 Bland JM, Altman DG. Statistical methods for assessing agreement between two methods of clinical measurement. Lancet 1986;1:307-10.

15 Bussmann JB, Stam HJ. Techniques for measurement and assessment of mobility in rehabilitation: a theoretical approach. Clin Rehabil 1998; 12:455-64

16 Cronbach L, Meehl P. Construct validity in psychological tests. Psychol Bull 1955:52:281-302

17 LaPorte RE, Montoye HJ, Caspersen CJ. Assessment of physical activity in epidemiologic research: problems and prospects. Public Health Rep 1985; 100:131-46.

18 Wiles CM, Newcombe RG, Fuller KJ, et al. Use of videotape to assess mobility in a controlled randomized crossover trial of physiotherapy in chronic multiple sclerosis. Clin Rehabil 2003;17:256-63. 